\title{
Bromodomain-containing protein 7 contributes to myocardial infarction-induced myocardial injury through activating Wnt/ $\beta$-catenin signaling
}

\author{
Xiaosa Chi ${ }^{1}$, Liang Shan ${ }^{2}$, Yi Hu ${ }^{1}$, Yuzhu Zhang', Yongjun Mao ${ }^{1}$, Xiaomeng Wang ${ }^{1}$ \\ ${ }^{1}$ Department of Geriatrics, Affiliated Hospital of Qingdao University, Qingdao, China; ${ }^{2}$ Department of Intensive Care Medicine, Affiliated Hospital \\ of Qingdao University, Qingdao, China \\ Contributions: (I) Conception and design: X Chi, Y Mao, X Wang; (II) Administrative support: X Wang; (III) Provision of study materials or patients: \\ X Chi, L Shan, Y Hu, Y Zhang; (IV) Collection and assembly of data: X Chi, L Shan, Y Hu, Y Zhang; (V) Data analysis and interpretation: Y Mao, \\ X Wang; (VI) Manuscript writing: All authors; (VII) Final approval of manuscript: All authors. \\ Correspondence to: Yongjun Mao; Xiaomeng Wang. Department of Geriatrics, Affiliated Hospital of Qingdao University, 59 Haier Road, Qingdao \\ 266100, China. Email: mmc168@126.com; qdwxm310@163.com.
}

Background Myocardial infarction (MI) is one of the most common cardiovascular diseases, inducing
severe myocardial injury and leading to high mortality. Bromodomain-containing protein 7 (BRD7), a
member of bromodomain-containing protein family, is involved in multiple cellular processes, such as cell
cycle, transcriptional regulation, and chromatin remodeling, but the functions of BRD7 in regulating MI-
associated myocardial injury are still obscure. In this work, we investigated the effect of BRD7 on MI-
induced myocardial injury in vitro and in vivo.

Methods: The MI model was established by ligating the left anterior descending coronary artery (LAD) of rats which were then injected with BRD7 short hairpin RNA (shRNA). The rat H9C2 cardiomyocytes were treated with hypoxia and injected with BRD7 shRNA. The expression of BRD7 in MI rat model, and hypoxia-treated H9C2 cells was detected by quantitative polymerase chain reaction (qPCR), western blot, and immunohistochemical staining. The effect of BRD7 was analyzed using western blot, terminal deoxynucleotidyl transferase dUTP nick end labeling (TUNEL) assay, echocardiography, and flow cytometry analysis. The expressions of $\mathrm{Wnt} / \beta$-catenin signaling relative proteins were determined by western blot.

Results: Significantly, BRD7 was highly expressed in MI patients, MI rat models, and hypoxia treated rat H9C2 cardiomyocytes. Echocardiography analysis demonstrated that the left ventricular ejection fraction (LVEF) and left ventricular fraction shortening (LVFS) were repressed in the MI rats relative to sham group rats, while the silencing of BRD7 rescued the dysfunction in the model. We also found that BRD7 silencing reduced cardiomyocyte apoptosis in both MI rats and H9C2 cells under the treatment of hypoxia. BRD7 silencing inhibited the activation of $\mathrm{Wnt} / \beta$-catenin signaling in $\mathrm{H} 9 \mathrm{C} 2$ cells under the treatment of hypoxia. Moreover, Wnt agonist BML294 reversed the anti-apoptosis effect of BRD7 silencing in hypoxia-induced H9C2 cells.

Conclusions: Collectively, we concluded that BRD7 contributed to MI-induced myocardial injury through activating $\mathrm{Wnt} / \beta$-catenin signaling. Targeting BRD7 may become a promising therapeutic strategy for the treatment of MI-induced myocardial injury.

Keywords: Myocardial infarction (MI); myocardial injury; apoptosis; BRD7; Wnt/ $\beta$-catenin signaling

Submitted Aug 12, 2021. Accepted for publication Sep 24, 2021.

doi: 10.21037/apm-21-2433

View this article at: https://dx.doi.org/10.21037/apm-21-2433 


\section{Introduction}

Cardiovascular diseases have the highest incidence and mortality around the world (1). Among various cardiovascular diseases, myocardial infarction (MI) is the major cause of death, leading to great loss and burden of both productivity and economy (2). MI usually initiates with complete coronary artery occlusion because of the rupture of an atherosclerotic plaque and the subsequent thrombotic process (3). The cause of MI involves inadequate blood and oxygen supply, and the subsequent death of myocardial cells (2). Current therapy for MI is mainly reperfusion strategies such as coronary thrombolysis and heart bypass surgery, and therapeutic drugs such as calcium antagonists and adrenoceptor blockers $(4,5)$. However, the progression of MI remains irreversible, and the reperfusion process after ischemia may lead to severe pathological damage of body tissues such as heart and brain $(2,6)$. Therefore, exploring the detailed pathological mechanisms and effective therapeutic targets has been a focus area for MI research.

It has been shown that Wnt signaling is an essential regulator during cardiomyogenesis and cardiac activity postMI $(7,8)$. The Wnt signaling includes three major pathways characterized by altered downstream effectors including the canonical $\beta$-catenin, non-canonical planar cell polarity (PCP), and $\mathrm{Ca}^{2+}$ signaling (9). Among which, $\beta$-catenin plays a central role in Wnt signaling transduction. Upon activation of $\mathrm{Wnt}, \beta$-catenin is able to translocate into nuclei and thereby facilitate the function of transcriptional factors and gene expression $(9,10)$. Recent studies have indicated a promising therapeutic effect of blocking of Wnt/ $\beta$-catenin signaling transduction on MI model. For instance, pyrvinium, the Food and Drug Administration (FDA)approved Wnt inhibitor for stabilizing $\beta$-catenin, could increase the portion of active cardiomyocytes around the infract area (11). It has also been indicated that ICG-001, the specific inhibitor targeting the function of $\beta$-catenin, could enhance epicardial progenitor differentiation, which contributes to improvement of myocardial function (12).

The expression of BRD7 has been detected in multiple tissues, including heart, at all life stages of life from infancy to adulthood $(13,14)$. Accumulating evidences have revealed the multifunctional roles of BRD7 in several biological processes such as ferroptosis of hepatic stellate cells and cancer metastasis, by regulating gene expression and cell growth $(15,16)$. Moreover, it is reported that inhibition of BRD7 protects against hyperglycaemia-induced cardiomyocyte apoptosis by inhibiting ER stress signalling pathway (17). However, the function of BRD7 in the pathogenesis of MI-induced myocardial injury is unclear, and explored firstly in our study. We discovered an elevated level of BRD7 in patients with MI, and identified its downstream $\mathrm{Wnt} / \beta$-catenin signaling by using in silico analysis and experimental testification. Our work proposed BRD7 as a promising target for MI therapy. We present the following article in accordance with the ARRIVE reporting checklist (available at https://dx.doi. org/10.21037/apm-21-2433).

\section{Methods}

\section{Materials}

The adenovirus vector containing shRNA targeting BRD7 (Ad-shBRD7) and the empty vector (Ad-shNC) were designed by and brought from QIAGEN (Hilden, Germany). Terminal deoxynucleotidyl transferase dUTP nick end labeling (TUNEL) assay kit and apoptosis detection kit was purchased from Thermo Fisher Scientific (Waltham, MA, USA). Antibodies against BRD7, Bax, Cleaved caspase-3, Bcl-2, Wnt, $\beta$-catenin, p-GSK-3 $\beta$, GSK-3, and glyceraldehyde 3-phosphate dehydrogenase (GAPDH) were ordered from R \& D system (Minneapolis, MN, USA).

We purchased $\mathrm{H}_{2} \mathrm{O}_{2}$ was from Sigma-Aldrich (St. Louis, MO, USA). The Wnt agonist BML-284 was ordered from AbMole BioScience (Houston, TX, USA).

\section{In silico analysis}

We downloaded a GSE141512 dataset (https://www.ncbi. nlm.nih.gov/geo/query/acc. . gi ?acc=GSE141512) from the Gene Expression Omnibus (GEO) database to determine the mRNA level of BRD7. The GSE141512 dataset consists of transcriptome information of peripheral blood mononuclear cells collected from MI patients and healthy donors. The analysis was performed on Affymetrix Human Transcriptome Array 2.0 platform (http://www.affymetrix. com/). We used the Comparative Toxicogenomics Database (CTD, https://ctdbase.org/) to screen potential MI-related signaling pathways, and the Search Tool for the Retrieval of Interacting Genes (STRING; http://string.embl.de/) database to select BRD7-associated signaling. The study was conducted in accordance with the Declaration of 
Helsinki (as revised in 2013).

\section{Animal model}

Sprague-Dawley rats $(250-300 \mathrm{~g}, 8$-week-old) that fit specific pathogen-free (SPF) standard were ordered from Vital River Laboratory (Beijing, China). The MI model was established by ligating the left anterior descending coronary artery (LAD) of rats. In brief, the rats were anesthetized and placed in a ventilator with heating plate. The chest was opened to expose the heart, and the LAD was ligated by an 6-0 suture. The successful blood occlusion was verified by ST-segment elevation monitored by electrocardiography. Sham group rats received the same operation but not the LAD ligation. For treatment of rats, adenovirus vector $\left(1 \times 10^{9} \mathrm{TU} / \mathrm{mL}, 10 \mu \mathrm{L}\right)$ carrying shBRD7 or shNC, were injected at 3 injection sites in the infarct section of the heart immediately post $\mathrm{MI}$ induction. At 4 weeks after treatment, the rats were anesthetized, and their cardiac function was observed by transthoracic echocardiography in an ultrasound Vevo system (Visual Sonics Company, Toronto, ON, Canada) following the manufacturer's instructions. The left ventricular ejection fraction (LVEF\%) and fractional shortening (LVFS\%) were captured and calculated at the frequency of $30 \mathrm{MHz}$. The hearts were then isolated and retained for further experiments. The rats were randomly assigned to five groups (8 rats in each group): Control group (Control), Sham group (Sham-operated group), MI group (LAD model group), $\mathrm{MI}+\mathrm{Ad}-\mathrm{shNC}$ group (LAD with Ad-shNC) and MI + Ad-shBRD7 group (LAD with Ad-shBRD7). Animal experiments were performed under a project license (No. QYFYWZLL26551) granted by the Animal Ethics Committee of the Affiliated Hospital of Qingdao University, in compliance with the Affiliated Hospital of Qingdao University guidelines for the care and use of animals.

\section{Immunohistochemical staining and TUNEL staining assay}

The heart tissues isolated from rats were paraffin-embedded and cut into $4 \mu \mathrm{m}$-thick slices. The tissue sections were then deparaffinized and rehydrated, blocked by $2 \% \mathrm{H}_{2} \mathrm{O}_{2}$ solution, permeabilized by $0.1 \%$ Triton $\mathrm{X}-100$, hatched in $5 \%$ bovine serum albumin (BSA), then labelled with primary anti-BRD7 antibody and subsequent horseradish peroxidase (HRP)-conjugated secondary antibody. The nucleus was visualized by staining with hematoxylin. For detection of apoptotic cells in heart tissues, we conducted terminal deoxynucleotidyl transferase-mediated nick-end labeling (TUNEL) assay following the manufacturer's description. In brief, the apoptotic cells were stained with TUNEL and nuclei were stained with 4',6-diamidino-2phenylindole (DAPI). Then, five random sections of each slice were photographed by microscope (Olympus, Tokyo, Japan), and the portion of positive staining was calculated by Image J software (https://imagej.nih.gov.ij/).

\section{Cell culture and bypoxia model}

Rat H9C2 cardiomyoblast cell line was obtained from American Type culture collection (ATCC, Manassas, VA, USA), and maintained in Dulbecco's modified Eagle's medium (DMEM) supplemented with 10\% fetal bovine serum (FBS; Gibco, Amarillo, TX, USA), $100 \mu \mathrm{g} / \mathrm{mL}$ streptomycin, and $100 \mathrm{U} / \mathrm{mL}$ penicillin. The hypoxia of $\mathrm{H} 9 \mathrm{C} 2$ cells were performed by using $\mathrm{H}_{2} \mathrm{O}_{2}$. In short, $\mathrm{H} 9 \mathrm{C} 2$ cells were placed in 6 -well plates $\left(1 \times 10^{5}\right.$ cells/well $)$ overnight and treated with $200 \mu \mathrm{M} \mathrm{H}_{2} \mathrm{O}_{2}$ for $4 \mathrm{~h}$. The cells were then digested and collected for indicated experiment.

\section{Apoptosis}

The apoptosis of H9C2 cells was measured by apoptosis detection kit under instruction of the manufacturer's protocol. In short, H9C2 cells were infected with AdshBRD7 or Ad-shNC in hypoxic or normoxic conditions, and treated with BML294 at $1 \mu \mathrm{M}$ for $48 \mathrm{~h}$. Next, the cells were collected by digestion and centrifuged, resuspended in binding buffer, and stained with Annexin-FITC and propidium iodide (PI) solution in the dark. The samples were detected by a flow cytometer (Becton, Dickinson, and Co. Biosciences, Franklin Lakes, NJ, USA) immediately.

\section{Quantitative real-time PCR}

RNA isolation from heat tissues and H9C2 cells was performed using Trizol reagent (Sigma, USA). The quantification of BRD7 was determined by using a SuperScript IV One-Step RT-PCR System (Thermo, USA) following the manufacturer's instruction and calculated by $2^{-\Delta \Delta \mathrm{Ct}}$ method. We used GAPDH as the internal control to normalize expression of BRD7. The primers of BRD7 and GAPDH were as follows: BRD7: forward, 5'-CGACCATGACAAACACAAGGA-3', and reverse, 5'-GGTCTCGATCCCGCTTCTTT-3'; GAPDH: forward, 5'-CCGCATCTTCTTGTGCAGTG-3', and 
reverse, 5'-CGATACGGCCAAATCCGTT-3'.

\section{Western blotting}

The H9c2 cells and heart tissues were lysed in radioimmunoprecipitation assay (RIPA) buffer (Sigma) to extract total protein. The protein was quantified by using bicinchoninic acid (BCA) kit (Thermo), separated by sodium dodecyl sulfate-polyacrylamide gel electrophoresis (SDS-PAGE), and then blotted to polyvinylidene difluoride (PVDF) membranes. The protein bands were blocked by fast blocking reagent (Millipore, Darmstadt, Germany), hatched with specific primary antibodies (anti-BRD7, antiBax, anti-Cleaved caspase- 3 , anti-Bcl-2, anti-Wnt, anti- $\beta$ catenin, anti-p-GSK-3 $\beta$, anti-GSK-3, and anti-GAPDH) in a $4{ }^{\circ} \mathrm{C}$ refrigerator for 1 night. The next day, the blots were visualized by interacting with HRP-conjugated secondary anti-rabbit or anti-mouse antibody at room temperature for $1 \mathrm{~h}$, followed by electrochemiluminescence (ECL) incubation. The visualized bands were captured by an E-Gel Imager (Invitrogen, Carlsbad, CA, USA). The relative levels of targeted proteins were quantified by grey intensity and normalized to GAPDH.

\section{Statistical analysis}

The data were shown as the means \pm SD of three independent experiments. Student's $t$-test and one-way analysis of variance (ANOVA) were performed to check the statistical difference between two or more groups by the SPSS software (IBM Corp., Chicago, IL, USA). An evaluated value with $\mathrm{P}<0.05$ was set as statistically significant.

\section{Results}

The expression of BRD7 was enhanced in MI patients, MI rat models, and hypoxia treated $\mathrm{H} 9 \mathrm{C} 2 \mathrm{cells}$

To determine the association of BRD7 with MI, we detected the expression of BRD7 in MI patients, MI rat model, and hypoxia-treated rat $\mathrm{H} 9 \mathrm{C} 2$ cardiomyocytes. Significantly, the presence of BRD7 was increased in the clinical MI samples compared with the healthy cases (Figure 1A). Similarly, the protein levels of BRD7 were enhanced in MI rats relative to those in the sham group (Figure $1 B$ ). In addition, the messenger RNA (mRNA) expression of BRD7 was induced in MI rats relative to the sham group (Figure 1C). Besides, the accumulation of BRD7 was higher in MI rats than that in the sham group (Figure 1D). As expected, the protein (Figure $1 E$ ) and mRNA levels (Figure $1 F$ ) of BRD7 were upregulated in $\mathrm{H} 9 \mathrm{C} 2$ cells under the treatment of hypoxia compared with the control H9C2 cells. These results imply that BRD7 is positively correlated with MI.

\section{Silencing of BRD7 attenuated myocardial dysfunction in MI mice}

To verify the impact of BRD7 on MI-induced myocardial dysfunction, we established an MI rat model and those rats were injected with control short hairpin RNA (shRNA) or BRD7 shRNA. The mRNA (Figure $2 A$ ) and protein (Figure $2 B$ ) expression of BRD7 was increased in the MI rat relative to the sham group and the depletion of BRD7 by shRNA repressed the BRD7 expression compared with the control shRNA. The echocardiography analysis indicated that the left ventricular ejection fraction (LVEF) and left ventricular fraction shortening (LVFS) were attenuated in the MI rats relative to the sham group rats, while the silencing of BRD7 rescued the function of LVEF and LVFS in the model (Figure $2 C-2 E$ ), suggesting that the silencing of BRD7 attenuates myocardial dysfunction in MI mice.

\section{Knockdown of BRD7 relieved myocardial apoptosis in MI mice}

Given that myocardial apoptosis is a crucial characteristic of MI-induced myocardial injury, we became interested in the impact of BRD7 depletion on this phenotype. Terminal deoxynucleotidyl transferase dUTP nick end labeling TUNEL staining revealed that the TUNEL-positive cells were increased in the MI rats relative to sham group rats and the silencing of BRD7 attenuated this phenotype in the model (Figure 3A). Consistently, Bax and cleaved caspase-3 expression was enhanced but Bcl-2 expression was reduced in the MI rats, while the BRD7 knockdown reversed this result in the model (Figure $3 B-3 E$ ), indicating that the knockdown of BRD7 relieves myocardial apoptosis in MI mice.

\section{Depletion of BRD7 repressed hypoxia-induced cardiomyocyte apoptosis in vitro}

Next, we further confirmed the impact of BRD7 on cardiomyocyte apoptosis in hypoxia treated H9C2 cells. We validated that hypoxia-induced BRD7 mRNA 

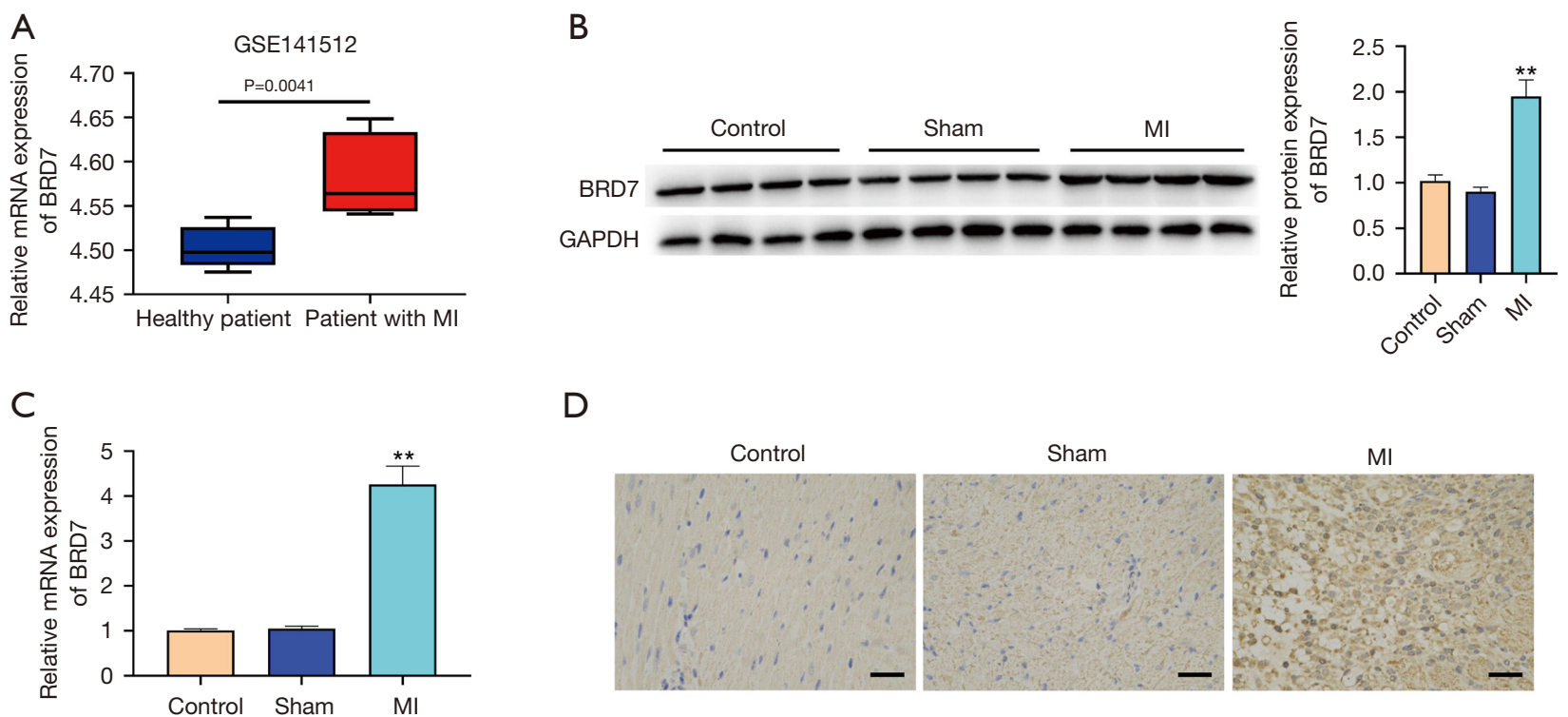

D
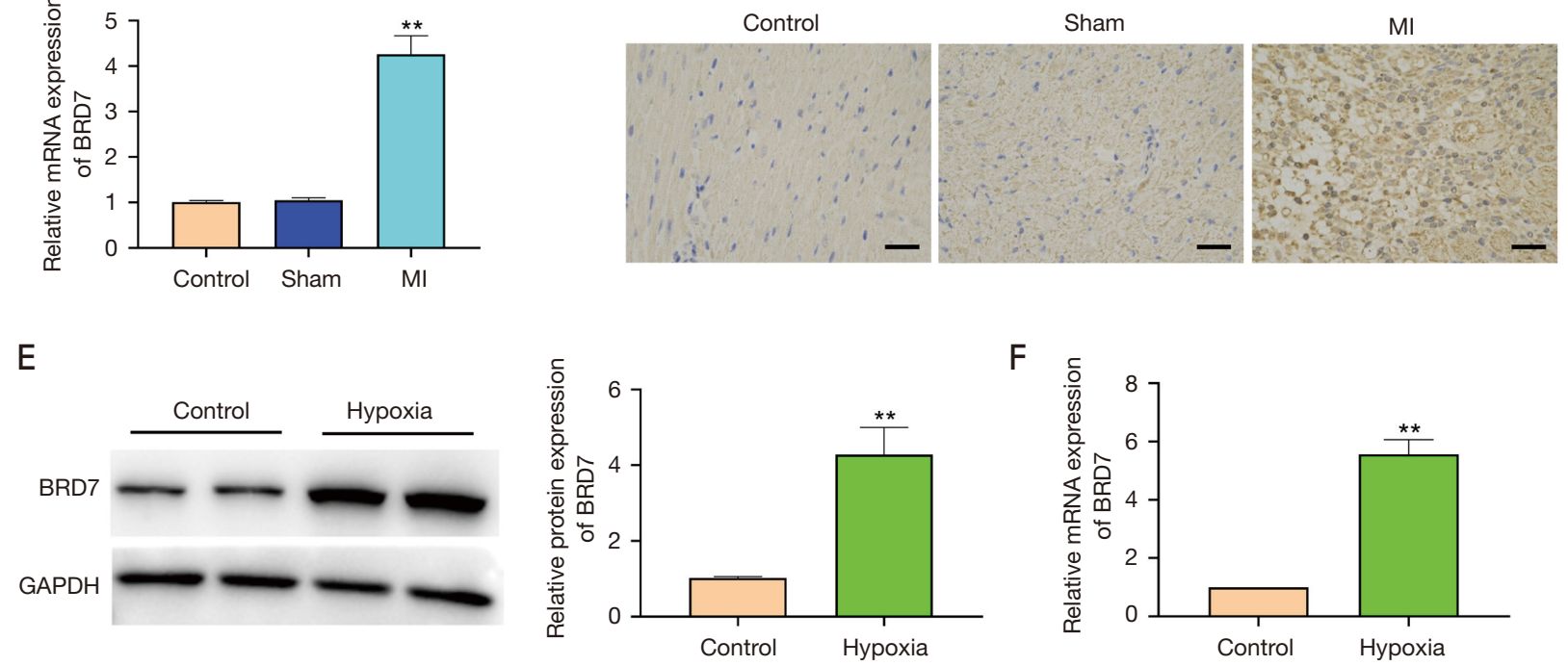

Figure 1 The expression of BRD7 is enhanced in MI patients, MI rat models, and hypoxia treated H9C2 cells. (A) The expression of BRD7 was analyzed in the healthy group and MI patients of GSE141512 from GEO database. (B) The MI model was established by ligating the $\mathrm{LAD}$ of rats. The protein expression of BRD7 was detected western blot analysis in the MI rat model. **, $\mathrm{P}<0.01$ vs. Sham group. (C) The mRNA expression of BRD7 was tested by qPCR in the MI rat model. **, $\mathrm{P}<0.01$ vs. Sham group. (D) The levels of BRD7 were determined by IHC (magnification 200x), brown yellow represents positive expression. (E) The protein expression of BRD7 was examined by Western blot analysis in rat $\mathrm{H} 9 \mathrm{C} 2$ cardiomyocytes or hypoxia-treated H9C2 cells. **, $\mathrm{P}<0.01$ vs. Control group. (F) The mRNA expression of BRD7 was assessed by qPCR in the MI rat model. **, $\mathrm{P}<0.01$ s. Control group. MI, myocardial infarction; GEO, Gene Expression Omnibus; LAD, left anterior descending coronary artery; mRNA, messenger RNA; qPCR, quantitative polymerase chain reaction; IHC, immunohistochemistry.

(Figure $4 A$ ) and protein expression (Figure $4 B$ ) was suppressed by the silencing of BRD7 by shRNA in H9C2 cells. The apoptosis of $\mathrm{H} 9 \mathrm{C} 2$ cells was induced by hypoxia and the depletion of BRD7 repressed this phenotype in the cells (Figure 4C). Consistently, Bax and cleaved caspase-3 expression was increased but Bcl-2 expression was decreased in hypoxia-treated $\mathrm{H} 9 \mathrm{C} 2$ cells, while the BRD7 knockdown reversed this result in the cells (Figure $4 D-4 G$ ), suggesting that the depletion of BRD7 represses hypoxia-induced cardiomyocyte apoptosis in vitro.

\section{BRD7 targeted Wnt/B-catenin signaling in cardiomyocytes}

To explore the potential mechanism of BRD7-meidtaed cardiomyocyte injury, we performed overlap analysis based on the MI-related pathways screened by CTD website and BRD7-related pathways screened by STRING website. The overlap analysis identified a total of 11 signaling pathways (Figure 5A) and the enrichment of these signaling pathways is shown in Figure 5B. Western blot analysis validated that the Wnt1 (Figure 5C,5D) and $\beta$-catenin (Figure 5C,5E) 
A

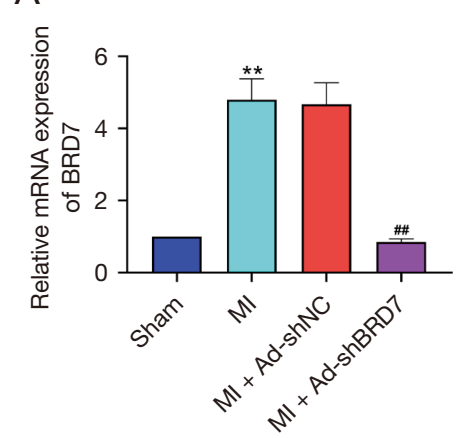

C

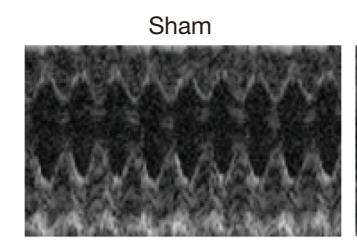

B

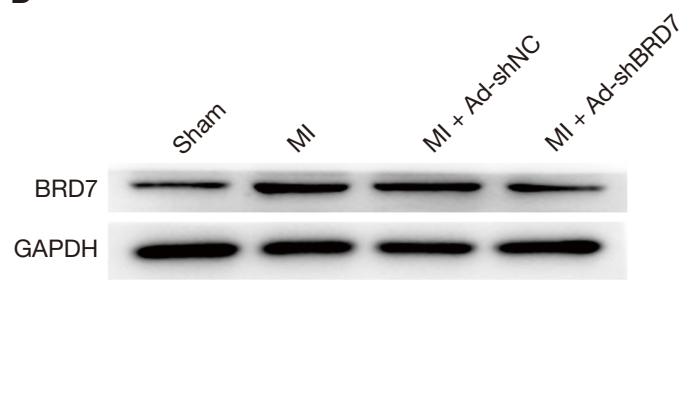

MI

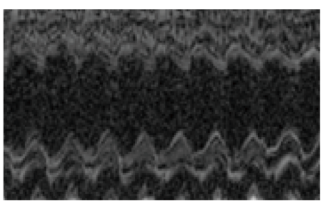

$\mathrm{Ml}+\mathrm{Ad}-\mathrm{shNC}$
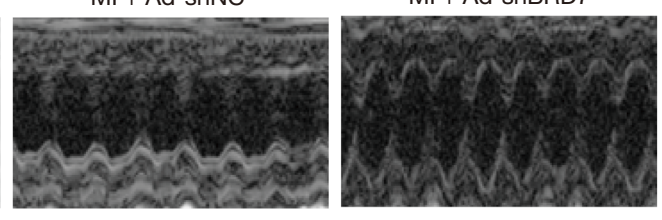

D

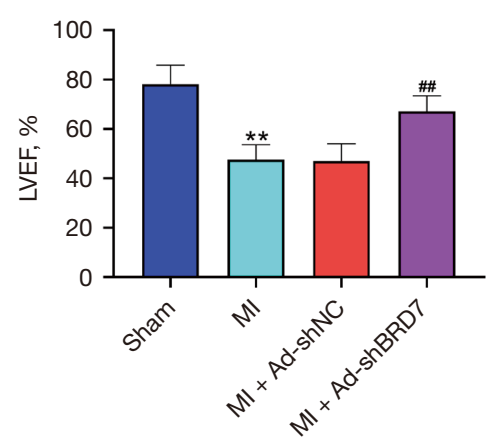

$E$

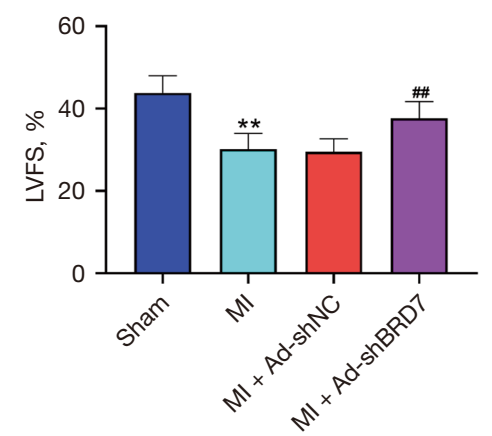

Figure 2 The silencing of BRD7 attenuates myocardial dysfunction in MI mice. (A-E) Adenovirus vector $\left(1 \times 10^{9} \mathrm{TU} / \mathrm{mL}, 10 \mu \mathrm{L}\right)$ carrying shBRD7 or shNC, were injected at 3 injection sites in the infarct section of heart immediately post MI induction. (A) The mRNA expression of BRD7 was determined by qPCR in the model. (B) The protein levels of BRD7 were detected by western blot in the model. (C-E) The

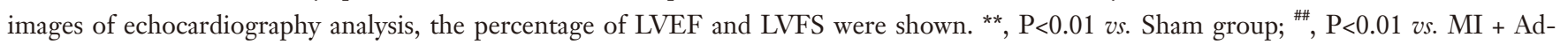
shNC group. MI, myocardial infarction; qPCR, quantitative polymerase chain reaction; mRNA, messenger RNA; LVEF, left ventricular ejection fraction; LVFS, left ventricular fractional shortening.

expression and GSK-3 $\beta$ phosphorylation (pGSK-3 $\beta$ )/GSK$3 \beta$ (Figure $5 C, 5 F$ ) were stimulated in the $\mathrm{H} 9 \mathrm{C} 2$ cells under the treatment of hypoxia, while the silencing of BRD7 using shRNA reversed this effect in the cells (Figure $5 C-5 F$ ), implying that BRD7 targets $\mathrm{Wnt} / \beta$-catenin signaling in cardiomyocytes.

\section{BRD7 contributed to bypoxia-induced cardiomyocyte apoptosis by targeting Wnt/ß-catenin signaling}

To confirm whether BRD7 modulates hypoxia-induced cardiomyocyte apoptosis by targeting $\mathrm{Wnt} / \beta$-catenin signaling, the hypoxia-stimulated $\mathrm{H} 9 \mathrm{C} 2$ cells were treated with BRD7 shRNA or co-treated with BRD7 shRNA and Wnt agonist BML294. The expression of Wnt1 (Figure 6A) and $\beta$-catenin (Figure 6B) and GSK$3 \beta$ phosphorylation (pGSK-3 $\beta$ ) (Figure $6 C$ ) inhibited by BRD7 shRNA were rescued by the treatment of BML294 in hypoxia-treated H9C2 cells (Figure 6A-6D). The expression of Bax (Figure $6 E, 6 F$ ) and cleaved caspase-3 (Figure 6E,6G) was reduced; but, Bcl-2 (Figure 6E,6H) expression was enhanced by BRD7 silencing in hypoxiatreated H9C2 cells and BML294 could reverse this effect (Figure 6E-6H). Consistently, hypoxia-treated H9C2 cell apoptosis was repressed by BRD7 depletion, while BML294 could rescue this phenotype (Figure 6I). Collectively, these 


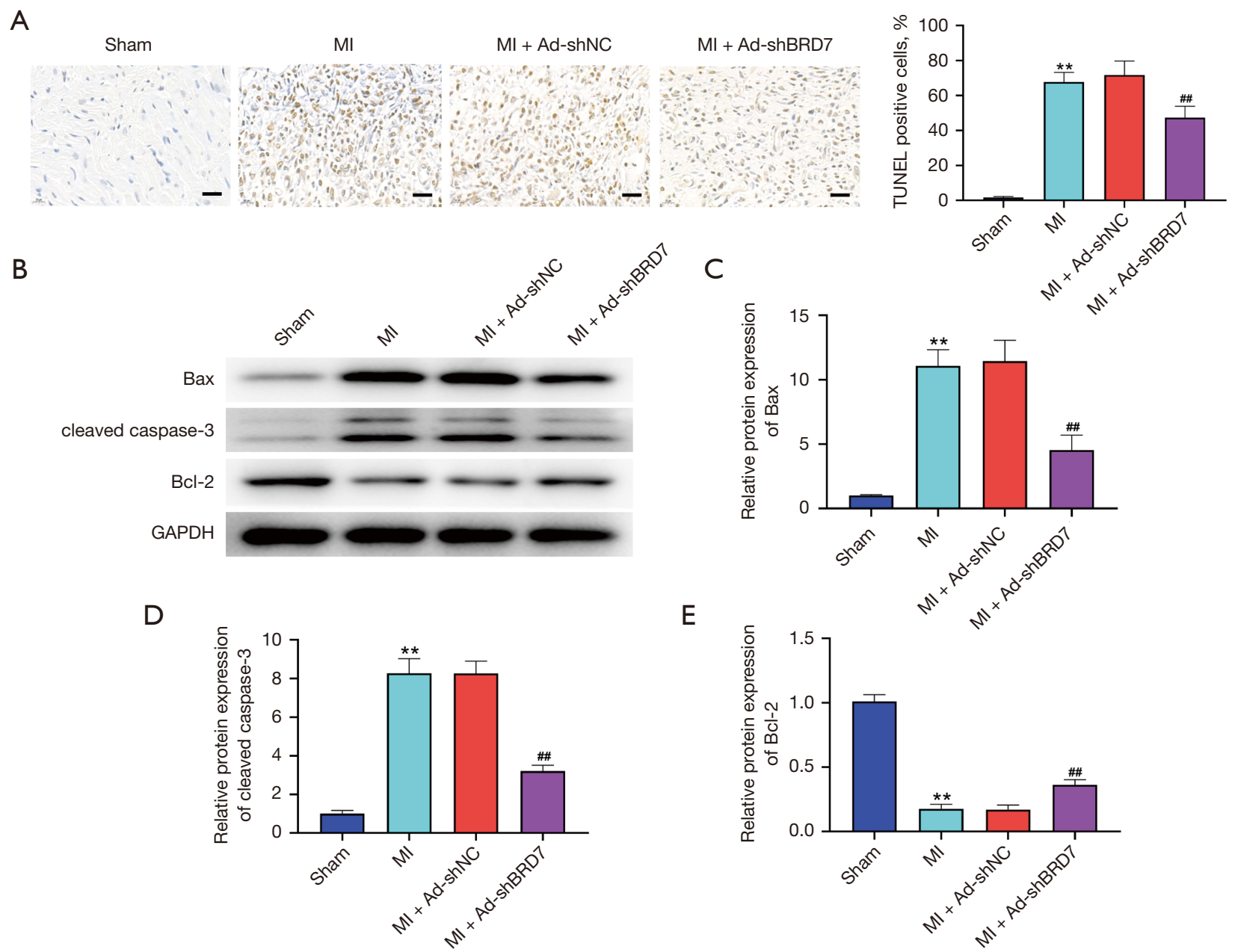

Figure 3 The knockdown of BRD7 relieves myocardial apoptosis in MI mice. (A) The apoptosis was detected by TUNEL analysis (TUNEL staining followed by DAPI staining) in the model $(\times 200)$, brown yellow represents positive expression. (B) The expression of Bax, cleaved caspase-3, and Bcl-2 was measured by western blot in the model. The results of the western blot were quantified by Image J software for Bax (C), cleaved caspase-3 (D), and Bcl-2 (E). **, $\mathrm{P}<0.01$ vs. Sham group; ${ }^{\#}, \mathrm{P}<0.01$ vs. MI + Ad-shNC group. MI, myocardial infarction; TUNEL, terminal deoxynucleotidyl transferase dUTP nick end labeling.

data indicate that BRD7 contributes to hypoxia-induced cardiomyocyte apoptosis by targeting $\mathrm{Wnt} / \beta$-catenin signaling.

\section{Discussion}

A prevalent cardiovascular disease, $\mathrm{MI}$ is characterized by myocardial ischemia and hypoxia; however, the molecular mechanisms underlying MI-induced myocardial injury are still poorly understood. In this research, we identified the crucial functions of BRD7 in affecting myocardial injury in vitro and in vivo.
Previous investigations have reported the abnormal expression and crucial roles of BRD7 in several disease models. It has been shown that BRD 7 regulates hyperglycemia-induced cardiac apoptosis by targeting endoplasmic reticulum stress signaling (17). In addition, BRD7 modulates the insulin signaling via enhancing GSK3 $\beta$ of phosphorylation in Wnt signaling (18). It also represses metastasis and invasion by conversely modulating YB1-related epithelial-mesenchymal transition in breast cancer (16). The mechanisms of MI-induced myocardial injury are complicated. For example, the knockdown of HIMF relieves MI ischemic injury through enhancing 

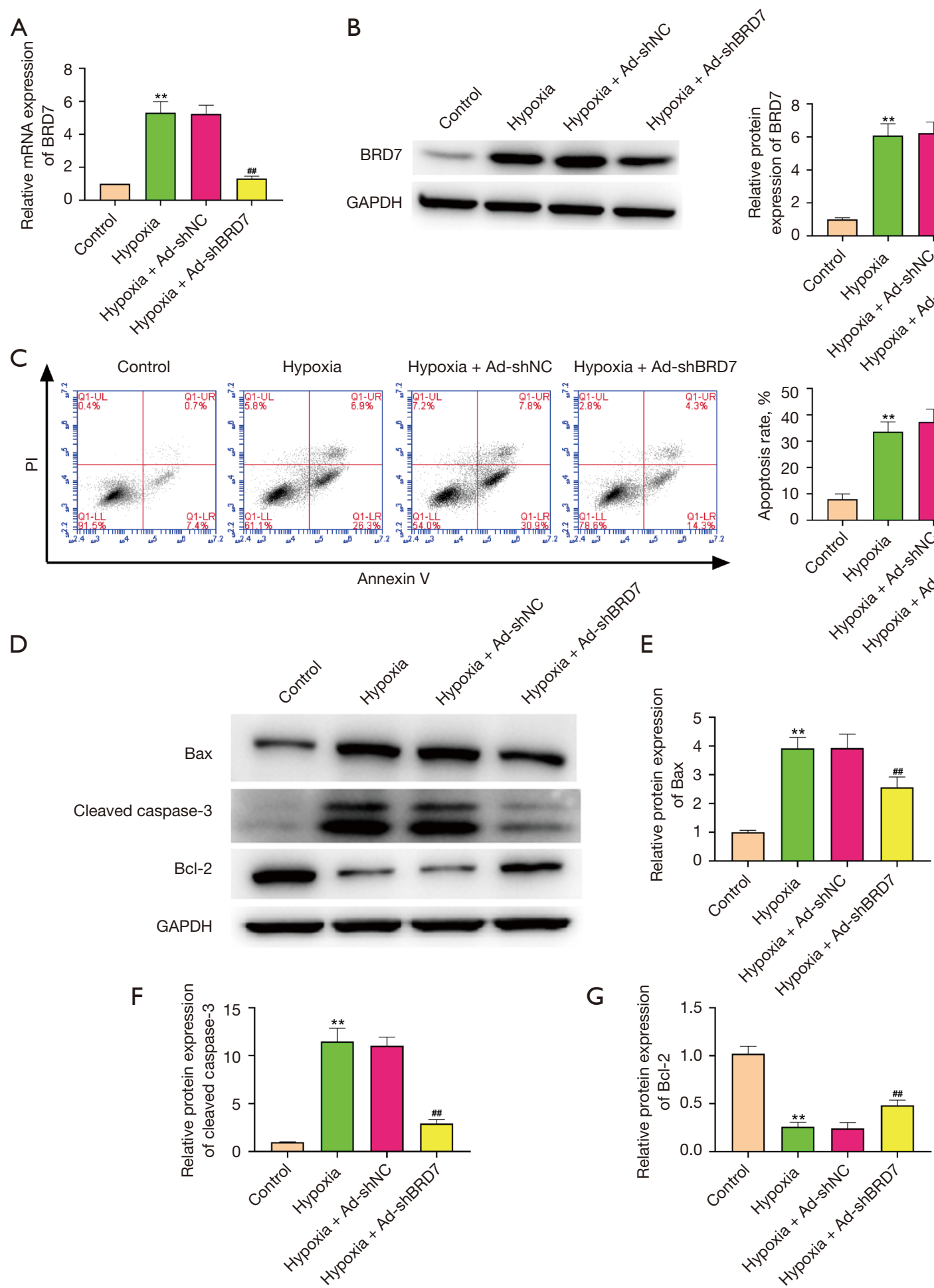

Figure 4 The depletion of BRD7 represses hypoxia-induced cardiomyocyte apoptosis in vitro. (A-G) Rat H9C2 cardiomyocytes were treated with hypoxia and BRD7 shRNA. (A) The mRNA expression of BRD7 was determined by qPCR in the cells. (B) The protein levels of BRD7 were detected by western blot in the cells. (C) The apoptosis was assessed by flow cytometry in the cells. (D-G) The expression of Bax, cleaved caspase-3, and Bcl-2 was measured by western blot in the cells. The results of the western blot were quantified by ImageJ software for Bax (E), cleaved caspase-3 (F), and Bcl-2 (G). **, $\mathrm{P}<0.01$ vs. Control group; ${ }^{* \#}, \mathrm{P}<0.01$ vs. hypoxia + Ad-shNC group. shRNA, short hairpin RNA; mRNA, messenger RNA; qPCR, quantitative polymerase chain reaction. 
A
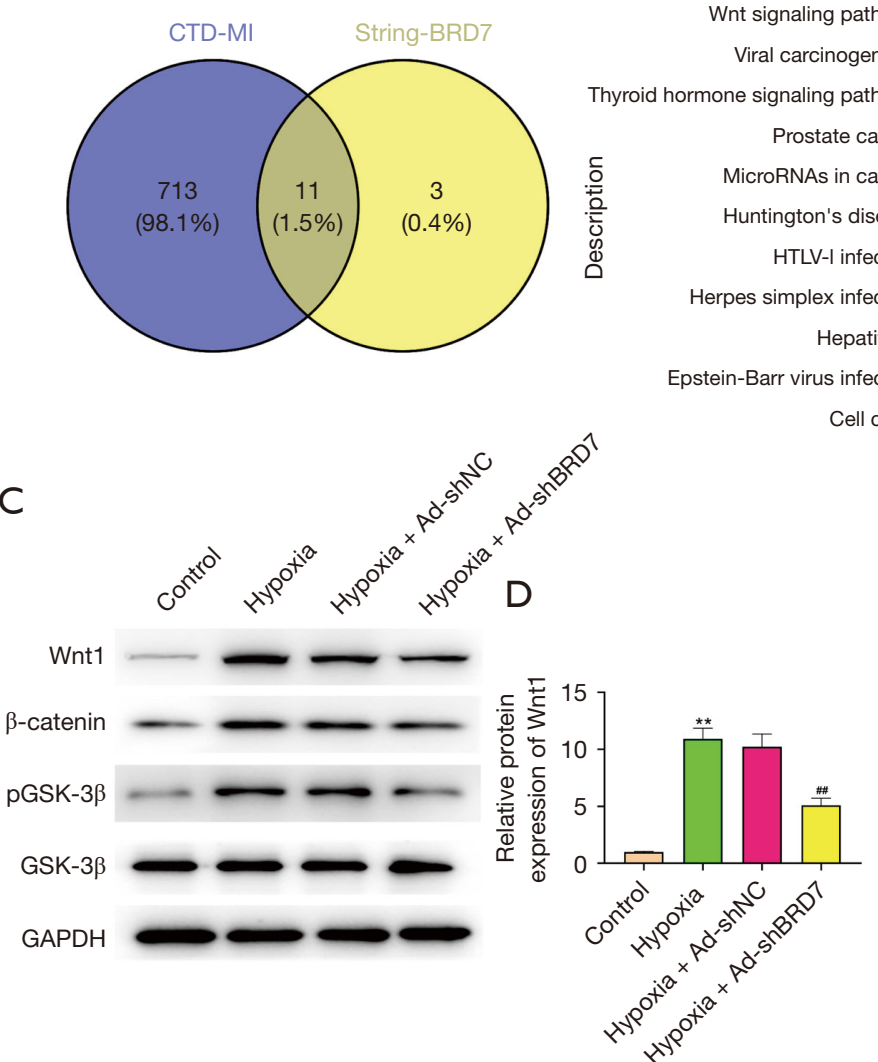

B

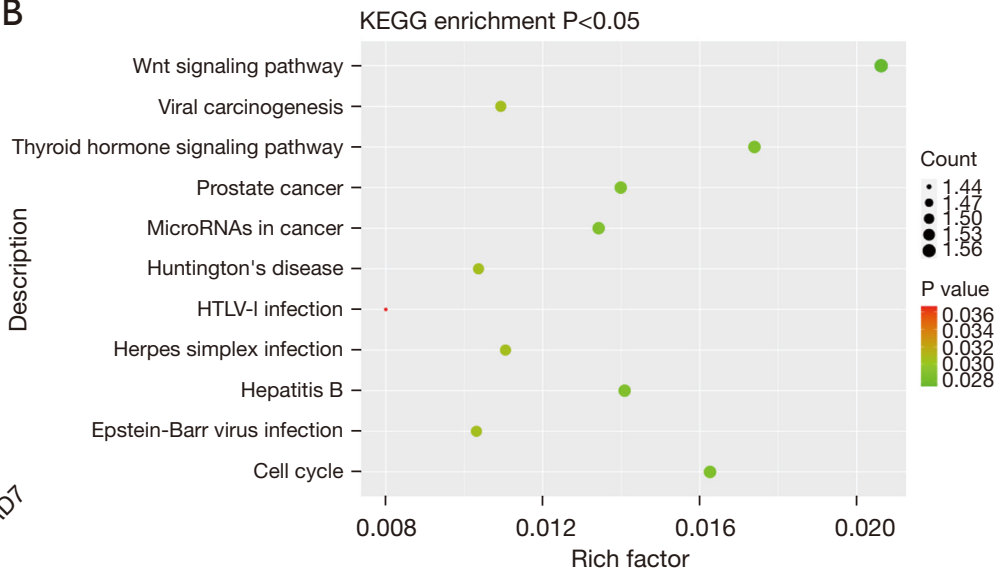

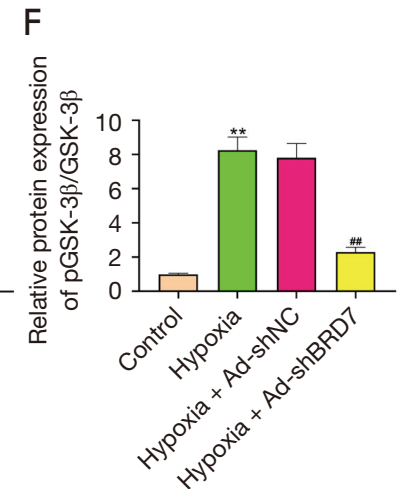

Figure 5 BRD7 targets Wnt/ $\beta$-catenin signaling in cardiomyocytes. (A) The overlap analysis between the myocardial infarction-related pathways screened by CTD website and BRD7-related pathways screened by STRING website. (B) A total of 11 signaling pathways were shown. (C-F) The expression of Wnt1, $\beta$-catenin, pGSK-3 $\beta$, and GSK-3 $\beta$ was measured by western blot in the cells. The results of the

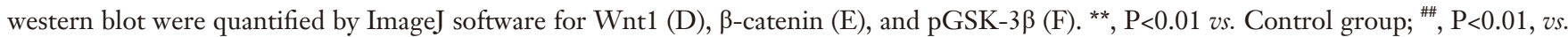
hypoxia + Ad-shNC group. CTD, Comparative Toxicogenomics Database; STRING, Search Tool for the Retrieval of Interacting Genes/ Proteins.

transformation of macrophages (19). The neuronal guidance molecule PMN-derived netrin-1 represses heart ischemia/reperfusion damage by targeting ADORA2B in MI (20). Human mesenchymal stem cell-delivered exosomal miR-543 contributes to angiogenesis after MI through modulation of COL4A1 (21). In this work, we revealed that BRD7 was enhanced in MI patients, $M I$ rat models, and hypoxia treated rat H9C2 cardiomyocytes. Echocardiography analysis confirmed that LVEF and LVFS were inhibited in the MI rats, while the silencing of BRD7 rescued the dysfunction. Apoptosis was increased in the MI rats and silencing of BRD7 attenuated this phenotype in the model. Hypoxia-induced apoptosis of H9C2 cells was repressed by BRD7 depletion. These results suggest that BRD7 can relieve MI-induced myocardial dysfunction and apoptosis in vitro and in vivo. Our data elucidate the new function of BRD7 in modulating MI-induced myocardial injury and enrich the knowledge of the understanding of the mechanisms of MI progression. The clinical values of BRD7 should be explored in future investigations.

The Wnt signaling has been recognized as a critical contributor to MI-induced myocardial injury. It has been reported that aldehyde dehydrogenase- 2 represses MIrelated cardiac fibrosis by the regulation of $\mathrm{Wnt} / \beta$-catenin signaling (22). Knockout of Wnt in macrophages enhances the function and remodeling in MI mouse model (23). The long non-coding RNA lncRNA-P21 represses apoptosis by targeting $\mathrm{Wnt} / \beta$-catenin signaling in acute $\mathrm{MI}$ rat model (24). The microRNA MiR-34a was shown to modulate MI-induced apoptosis by $\mathrm{Wnt} / \beta$-catenin signaling 
A

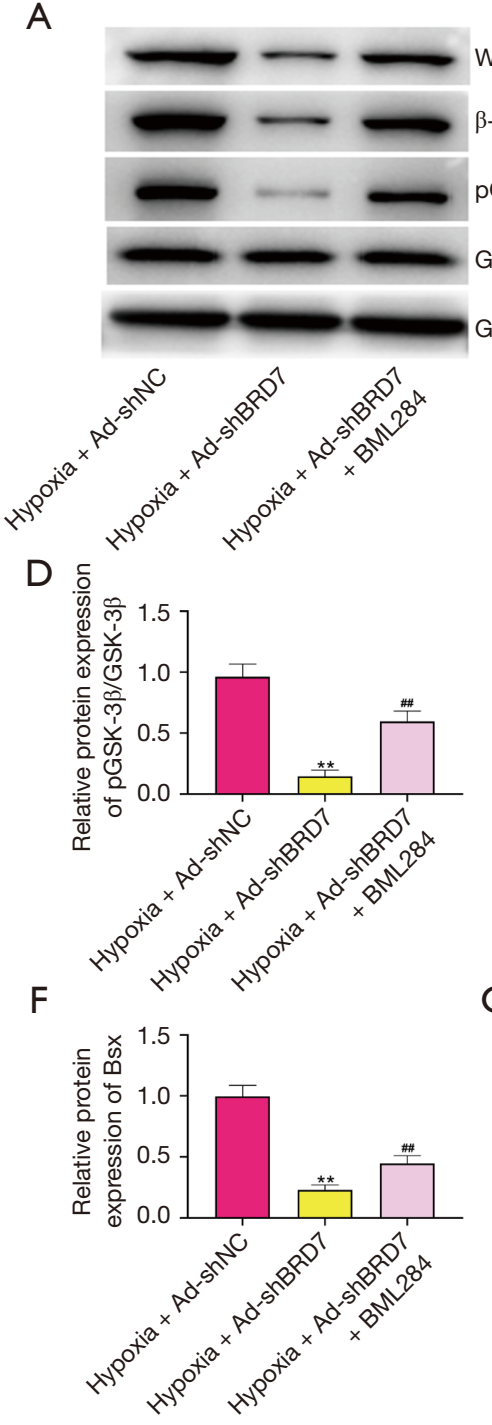

B

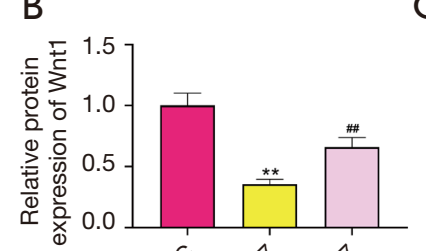

GSK-3ß

GAPDH

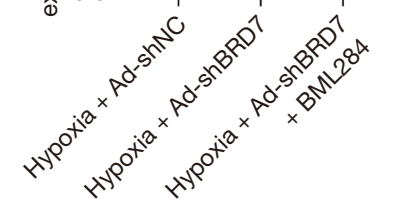

C .

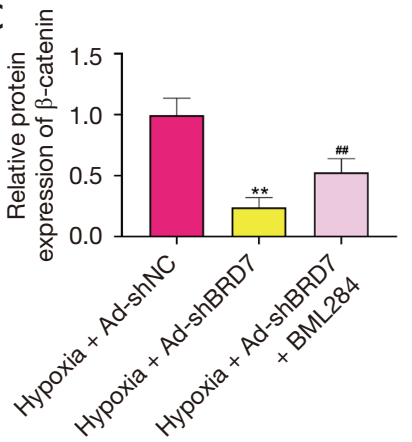

$E$
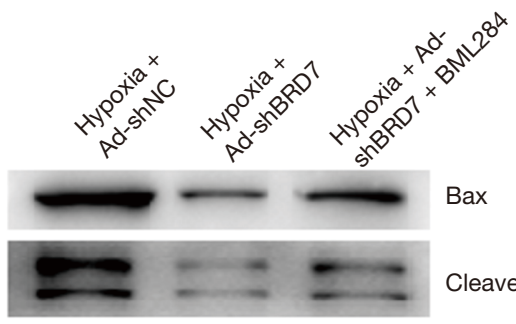

Cleaved caspase-3

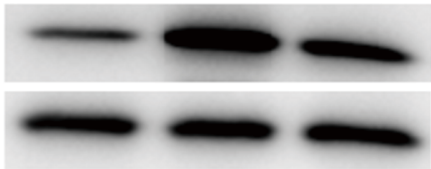

$\mathrm{Bcl}-2$

GAPDH
$\mathrm{G}$

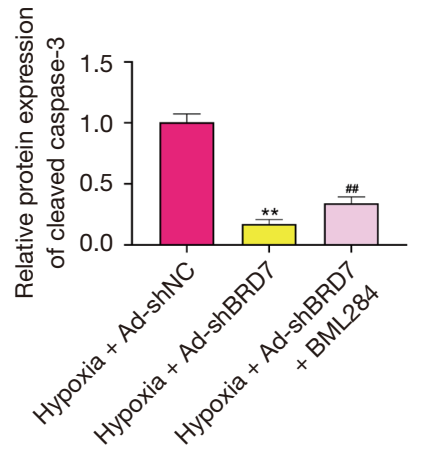

$\mathrm{H}$

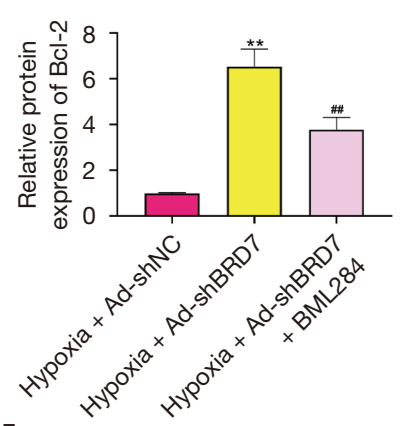

Hypoxia + Ad-shBRD7
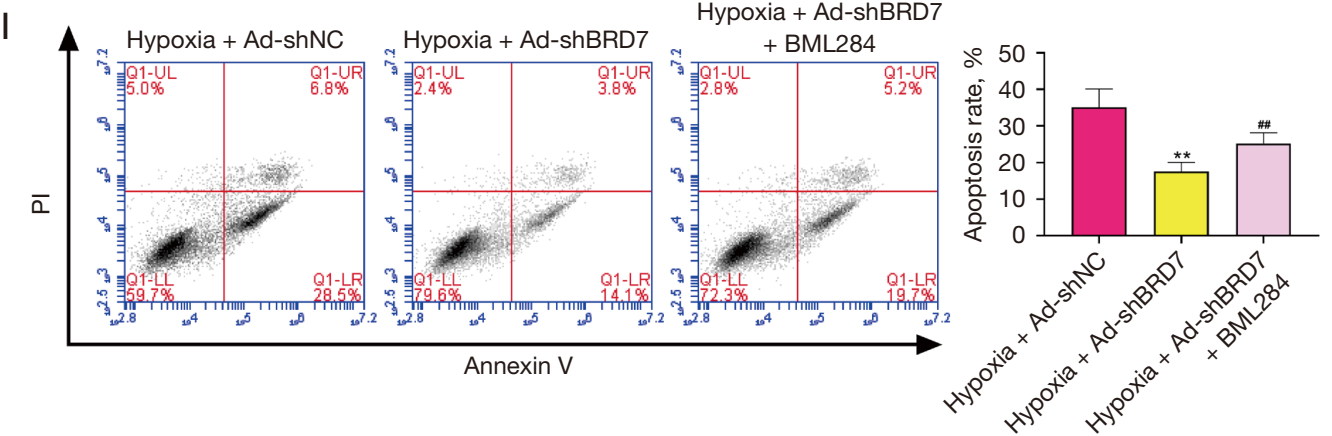

Figure 6 BRD7 contributes to hypoxia-induced cardiomyocyte apoptosis by targeting Wnt/ $\beta$-catenin signaling. (A-I) The hypoxia-stimulated H9C2 cells were treated with BRD7 shRNA or co-treated with BRD7 shRNA and BML294. (A-D) The expression of Wnt1, $\beta$-catenin, pGSK-3 $\beta$, and GSK-3 $\beta$ was measured by western blot in the cells. The results of western blot were quantified by ImageJ software for Wnt1 (B), $\beta$-catenin (C), and pGSK-3 $\beta$ (D). (E-H) The expression of Bax, cleaved caspase-3, and Bcl-2 was detected by western blot in the cells. The results of western blot were quantified by ImageJ software for Bax (F), cleaved caspase-3 (G), and Bcl-2 (H). (I) The apoptosis was assessed by flow cytometry in the cells. ${ }^{*}, \mathrm{P}<0.01$ vs. hypoxia + Ad-shNC group; ${ }^{* \#}, \mathrm{P}<0.01$ vs. hypoxia+Ad-shBRD7 group. shRNA, short hairpin RNA. 
in a rat model (25). Targeting Wnt signaling protects against MI-induced myocardial injury (26). In this study, we observed that Wnt1 and $\beta$-catenin expression and GSK-3 $\beta$ phosphorylation were enhanced in the $\mathrm{H} 9 \mathrm{C} 2$ cells under the treatment of hypoxia, while the silencing of BRD7 reversed this effect and Wnt agonist BML294 rescued the phenotype. Hypoxia-induced H9C2 cell apoptosis was suppressed by BRD7 depletion, while BML294 could reverse this alteration. These data imply that BRD7 contributes to hypoxia-induced cardiomyocyte apoptosis by targeting $W n t / \beta$-catenin signaling. Our data validate the crucial function of $\mathrm{Wnt} / \beta$-catenin signaling in $\mathrm{MI}$ induced myocardial injury and indicate a crucial mechanism involving BRD7 and Wnt/ $\beta$-catenin signaling. Whether BRD7 affects MI-induced myocardial injury by other signaling pathways requires confirmation though further research.

Taken together, we conclude that BRD7 contributed to MI-induced myocardial injury through activating Wnt/ $\beta$-catenin signaling. Targeting BRD7 may become a promising therapeutic strategy for the treatment of MIinduced myocardial injury.

\section{Acknowledgments}

Funding: This study was supported by Youth Foundation of National Natural Science Foundation of China: The role and mechanism of BRD7 in myocardial pyroptosis after myocardial ischemia in diabetes mellitus (Number: 82000268), and Youth Foundation of National Natural Science Foundation of China: Study on the effect of clock gene BMAL1 on drug resistance of temporal lobe epilepsy in rats through mTOR signaling pathway (Number: 81901321).

\section{Footnote}

Reporting Checklist: The authors have completed the ARRIVE reporting checklist. Available at https://dx.doi. org/10.21037/apm-21-2433

Data Sharing Statement: Available at https://dx.doi. org/10.21037/apm-21-2433

Conflicts of Interest: All authors have completed the ICMJE uniform disclosure form (available at https://dx.doi. org/10.21037/apm-21-2433). Dr. Chi reports this study was supported by Youth Foundation of National Natural
Science Foundation of China: Study on the effect of clock gene BMAL1 on drug resistance of temporal lobe epilepsy in rats through mTOR signaling pathway (Number: 81901321). Dr. Wang reports this study was supported by Youth Foundation of National Natural Science Foundation of China: The role and mechanism of BRD7 in myocardial pyroptosis after myocardial ischemia in diabetes mellitus (Number: 82000268). The authors have no other conflicts of interest to declare.

Ethical Statement: The authors are accountable for all aspects of the work in ensuring that questions related to the accuracy or integrity of any part of the work are appropriately investigated and resolved. Human Research in this study was conducted in accordance with the Declaration of Helsinki (as revised in 2013). Animal experiments were performed under a project license (No. QYFYWZLL26551) granted by the Animal Ethics Committee of the Affiliated Hospital of Qingdao University, in compliance with the Affiliated Hospital of Qingdao University guidelines for the care and use of animals.

Open Access Statement: This is an Open Access article distributed in accordance with the Creative Commons Attribution-NonCommercial-NoDerivs 4.0 International License (CC BY-NC-ND 4.0), which permits the noncommercial replication and distribution of the article with the strict proviso that no changes or edits are made and the original work is properly cited (including links to both the formal publication through the relevant DOI and the license). See: https://creativecommons.org/licenses/by-nc-nd/4.0/.

\section{References}

1. Carney RM, Freedland KE. Depression and coronary heart disease. Nat Rev Cardiol 2017;14:145-55.

2. Neri M, Riezzo I, Pascale N, et al. Ischemia/Reperfusion Injury following Acute Myocardial Infarction: A Critical Issue for Clinicians and Forensic Pathologists. Mediators Inflamm 2017;2017:7018393.

3. Badimon L, Vilahur G. Thrombosis formation on atherosclerotic lesions and plaque rupture. J Intern Med 2014;276:618-32.

4. Gerczuk PZ, Kloner RA. An update on cardioprotection: a review of the latest adjunctive therapies to limit myocardial infarction size in clinical trials. J Am Coll Cardiol 2012;59:969-78.

5. Wernly JA. Ischemia, reperfusion, and the role of surgery 
in the treatment of cardiogenic shock secondary to acute myocardial infarction: an interpretative review. J Surg Res 2004;117:6-21.

6. Li SY, Li ZX, He ZG, et al. Quantitative proteomics reveal the alterations in the spinal cord after myocardial ischemia-reperfusion injury in rats. Int $\mathrm{J}$ Mol Med 2019;44:1877-87.

7. Gessert S, Kühl M. The multiple phases and faces of wnt signaling during cardiac differentiation and development. Circ Res 2010;107:186-99.

8. Ozhan G, Weidinger G. Wnt/ $\beta$-catenin signaling in heart regeneration. Cell Regen 2015;4:3.

9. Duchartre Y, Kim YM, Kahn M. The Wnt signaling pathway in cancer. Crit Rev Oncol Hematol 2016;99:141-9.

10. Liu Y, Neogi A, Mani A. The role of Wnt signalling in development of coronary artery disease and its risk factors. Open Biol 2020;10:200128.

11. Saraswati S, Alfaro MP, Thorne CA, et al. Pyrvinium, a potent small molecule $\mathrm{Wnt}$ inhibitor, promotes wound repair and post-MI cardiac remodeling. PLoS One 2010;5:e15521.

12. Sasaki T, Hwang H, Nguyen C, et al. The small molecule Wnt signaling modulator ICG-001 improves contractile function in chronically infarcted rat myocardium. PLoS One 2013;8:e75010.

13. Fujisawa T, Filippakopoulos P. Functions of bromodomaincontaining proteins and their roles in homeostasis and cancer. Nat Rev Mol Cell Biol 2017;18:246-62.

14. Park SW, Lee JM. Emerging Roles of BRD7 in Pathophysiology. Int J Mol Sci 2020;21:7127.

15. Zhang Z, Guo M, Shen M, et al. The BRD7-P53SLC25A28 axis regulates ferroptosis in hepatic stellate cells. Redox Biol 2020;36:101619.

16. Niu W, Luo Y, Zhou Y, et al. BRD7 suppresses invasion and metastasis in breast cancer by negatively regulating YB1-induced epithelial-mesenchymal transition. J Exp Clin Cancer Res 2020;39:30.

17. Wang XM, Wang YC, Liu XJ, et al. BRD7 mediates

Cite this article as: Chi X, Shan L, Hu Y, Zhang Y, Mao Y, Wang X. Bromodomain-containing protein 7 contributes to myocardial infarction-induced myocardial injury through activating $W n t / \beta$-catenin signaling. Ann Palliat Med 2021;10(10):10756-10767. doi: 10.21037/apm-21-2433 hyperglycaemia-induced myocardial apoptosis via endoplasmic reticulum stress signalling pathway. J Cell Mol Med 2017;21:1094-105.

18. Golick L, Han Y, Kim Y, et al. BRD7 regulates the insulinsignaling pathway by increasing phosphorylation of GSK3ß. Cell Mol Life Sci 2018;75:1857-69.

19. Li Y, Dong M, Wang Q, et al. HIMF deletion ameliorates acute myocardial ischemic injury by promoting macrophage transformation to reparative subtype. Basic Res Cardiol 2021;116:30.

20. Li J, Conrad C, Mills TW, et al. PMN-derived netrin-1 attenuates cardiac ischemia-reperfusion injury via myeloid ADORA2B signaling. J Exp Med 2021;218:e20210008.

21. Yang M, Liu X, Jiang M, et al. miR-543 in human mesenchymal stem cell-derived exosomes promotes cardiac microvascular endothelial cell angiogenesis after myocardial infarction through COL4A1. IUBMB Life 2021;73:927-40.

22. Zhao X, Hua Y, Chen H, et al. Aldehyde dehydrogenase-2 protects against myocardial infarction-related cardiac fibrosis through modulation of the $W n t / \beta$-catenin signaling pathway. Ther Clin Risk Manag 2015;11:1371-81.

23. Palevski D, Levin-Kotler LP, Kain D, et al. Loss of Macrophage Wnt Secretion Improves Remodeling and Function After Myocardial Infarction in Mice. J Am Heart Assoc 2017;6:004387.

24. Liu FJ. LncRNA-P21 suppresses apoptosis of myocardial cells in rats with acute myocardial infarction via regulating Wnt/ $\beta$-catenin signaling pathway. Eur Rev Med Pharmacol Sci 2020;24:10078-85.

25. Li JH, Dai J, Han B, et al. MiR-34a regulates cell apoptosis after myocardial infarction in rats through the Wnt/ $\beta$-catenin signaling pathway. Eur Rev Med Pharmacol Sci 2019;23:2555-62.

26. Blankesteijn WM. Interventions in WNT Signaling to Induce Cardiomyocyte Proliferation: Crosstalk with Other Pathways. Mol Pharmacol 2020;97:90-101. 